\title{
Sustained localized reentry within the left atrial appendage as a mechanism of recurrent arrhythmia following atrial fibrillation ablation
}

\author{
JIN-CUN GUO $^{1 *}$, WEI-BIN HUANG ${ }^{1 *}$, FA-GUANG ZHOU ${ }^{1}$, JIANG HONG $^{2}$ and YAN WANG ${ }^{1}$ \\ ${ }^{1}$ Cardiology Department, Xiamen Cardiovascular Hospital Xiamen University, Xiamen, Fujian 361004; \\ ${ }^{2}$ Cardiology Department, Shanghai General Hospital, Shanghai 200080, P.R. China
}

Received October 19, 2017; Accepted April 13, 2018

DOI: $10.3892 /$ etm.2018.6243

\begin{abstract}
The current study investigated the electrophysiological characteristics and radiofrequency ablation in patients with localized reentry within the left atrial appendage during repeated ablation for recurrent atrial fibrillation (AF). A total of 76 patients (21 paroxysmal, 55 persistent) undergoing repeated catheter ablation for recurrent AF were enrolled in this study. Local reentry tachycardia within the left atrial appendage (LAA) was identified through combining activation and entrainment mapping. Left atriography was performed prior to radiofrequency ablation to identify the focus in the LAA. Three patients (1 paroxysmal, 2 persistent) with sustained atrial tachycardias (ATs) were identified during repeated ablation in this cohort. Combined activation and entrainment mapping were applied to localize the reentry. Postpacing interval-tachycardia cycle length differences were $<30 \mathrm{msec}$ at the possible site of reentry in varying segments with macro-reentry. This difference was only determined at the base of LAA for local reentry within the LAA. All 3 patients were free of atrial arrhythmias without any complications at the 6-month follow-up following the ablation in the LAA. Combining activation and entrainment mapping were necessary in approaching ATs within the LAA. Performing entrainments in opposite segments of possible loops were valuable in precluding macro-reentry. Focal ablation was safe and effective in this cohort. Therefore localized reentry within the LAA was not uncommon during repeat AF ablation. The present study may thus provide valuable information for clinicians to manage this type of arrhythmia.
\end{abstract}

Correspondence to: Dr Yan Wang, Cardiology Department, Xiamen Cardiovascular Hospital Xiamen University, 205 Hubin South Road, Xiamen, Fujian 361004, P.R. China

E-mail:wangyanwy9@163.com

${ }^{*}$ Contributed equally

Key words: localized reentry, atrial tachycardia, persistent atrial fibrillation, left atrial appendage ridge

\section{Introduction}

Arrhythmia recurrence is the most common electrophysiological complication following atrial fibrillation (AF) ablation, which occurs in up to $31 \%$ of patients undergoing this procedure and frequently requires repeated ablation procedures (1). Organized atrial tachycardias (ATs) developing during ablation of AF are common, particularly when extensive ablation strategies are employed (2-4). It is important to understand the underlying mechanisms of tachycardia for treatment and development of preventative strategies. Macro-reentrant arrhythmias are the most common forms of ATs, occurring in $34 \%$ of patients during ablation of persistent AF (5). The most frequent mechanisms are mitral annular and gap-associated macro-reentrant flutter (6,7). Previous studies suggested ridge-associated reentry of ATs and ATs utilizing the ligament of Marshall as possible mechanisms $(8,9)$. In addition to macro-reentrant tachycardia, localized reentry or focal ATs are relatively uncommon in patients following AF ablation (10-13). Foci do not occur randomly throughout the atria, but exhibit a tendency to cluster at characteristic anatomical locations. A total of $63 \%$ of ATs originate from the right atrium, while $37 \%$ are from the left atrium (13). Sites of origin within the right atrium include: The crista terminalis, the tricuspid annulus, the right atrial appendage, the ostium of the coronary sinus, and within perinodal locations (14). In the left atrium, the ostia of the pulmonary vein is a common location for foci, exhibiting smaller numbers at the superior aspect of the mitral annulus and body of the coronary sinus (15). Additionally, left atrial appendage (LAA) may be implicated in the maintenance of AF and ATs (16-19).

The aims of AT mapping are to identify foci or reentrant circuits of arrhythmia. The underlying mechanism of AT can be inferred from activation maps. However, low voltage and fractionated electrograms, commonly found in atria of redo-AF patients are prone to incorrect assignment of local activation time (20). Although tachycardia may be recognized by entrainment, this risks its transformation or termination, making local capture within scarred areas difficult (21). A combined strategy using activation mapping and entrainment is regularly utilized to overcome these individual limitations (20). 
Anatomic studies have demonstrated that the LA wall is usually smooth, with the pectinate muscles being contained mostly within the LAA (22-24). The response to RF applications in pectinate muscle areas differ from those in smooth muscle areas due to a lack of contact with the tissue and the thermal homeostatic effect of blood on the pectinate muscles (19). However, the LAA has a very thin wall and may be prone to perforation. Thus, caution should be exercised when LAA ablation is performed.

In the present study, local reentrant tachycardia within the LAA, either occurring spontaneously or induced during repeated ablation of AF, is described. The findings of the current study support the proposal that in selected patients with AF, LAA may serve as a source to harbor ATs and ablation may be performed successfully in these patients.

\section{Materials and methods}

Study population. A total of 76 patients (age range, 56-78 years, 22 female and 54 male) undergoing repeated catheter ablation (21 paroxysmal, 55 persistent) for symptomatic and drug-resistant recurrent AF in the Cardiology Department of Xiamen Cardiovascular Hospital (Xiamen, China) from July 2010 to April 2016. Baseline characteristics of the patients, including age, gender, left atrium size, left ventricular ejection fraction (EF) and presence of structural heart disease, were collected from medical records (Table I). Exclusion criteria were defined as follows: Patients $<18$ years old, patients with LA/LAA thrombus, patients with structural heart disease or unwillingness to participate. Antiarrhythmic medications, except amiodarone for $>4$ weeks, were discontinued for five half-lives prior to surgery. The current study was approved by the Institutional Ethics Committee of Xiamen Cardiovascular Hospital (Xiamen, China; XMCH-014-173) and was in compliance with national legislation and the Declaration of Helsinki guidelines. Patients provided written informed consent.

Electrophysiology study. Transesophageal echocardiography and cardiac computed tomography were performed in all the patients. Three femoral venous approaches were performed. A 6-Fr decapolar catheter (Bard Dynamic Tip; Boston Scientific Co., Marlborough, MA, USA) was positioned in the coronary sinus. Double transseptal access was achieved using an 8.5-Fr non-steerable sheath (SL1; St. Jude Medical, Inc., Saint Paul, MN, USA). Intravenous heparin (100 U/kg; Shenzhen Hai Purui Pharmaceutical Co., Ltd., Shenzhen, China) was administered to achieve an activated clotting time of 300-400 sec. A LassoNAV catheter (Biosense Webster, Inc., Irvine, CA, USA) was used for mapping and recording of pulmonary vein (PV) potentials. Surface and intracardiac electrocardiograms were recorded (Prucka CardioLab EP System; GE Healthcare, Chicago, IL, USA). Radiofrequency ablation was delivered using an open irrigated 7F, 3.5-mm-tip, pressure-sensitive ablation catheter (Biosense Webster, Inc.). Ablation lesions were delivered at 25-35 W, for 30-60 sec at $43^{\circ} \mathrm{C}$ using a temperature-controlled mode with an irrigation rate of $17-30 \mathrm{ml}$ per min. When ablating in the coronary sinus, $25 \mathrm{~W}$ for $25 \mathrm{sec}$ with an irrigating rate of $30 \mathrm{ml}$ was used.

A three-dimensional rendering of the left atrium (LA) was created from a computed tomography image of the LA and integrated with an electroanatomical map to guide ablation catheter navigation (CARTO MERGE; Biosense Webster, Inc.). Patients with paroxysmal AF underwent PV isolation (PVI) only. While a fixed ablation approach, consisting of circumferential PVI and three linear ablation lesion sets across the mitral isthmus, left atrial roof and cavotricuspid isthmus, was performed in patients with persistent AF. Introduction tests were then conducted in all patients. The introduction protocol was rapid pacing initiated with a cycle length of $250 \mathrm{msec}$ progressively shortening by 10 to $180 \mathrm{msec}$ or refractoriness. Inducibility was defined as atrial arrhythmia $>1 \mathrm{~min}$. Activation mapping using the CARTO mapping system and entrainment mapping were undertaken as diagnostic techniques to differentiate focal, localized reentry and macro-reentry ATs. For rapid distinction of left and right ATs, entrainment was performed at the high right atrium (RA), proximal coronary sinus (CS) and distal CS. Postpacing interval (PPI)-tachycardia cycle length (TCL) differences $<50 \mathrm{msec}$ at the high RA suggested RA reentrant circuits, while $>50$ msec suggested LA circuits. For LA circuits, PPI-TCL difference at the proximal and distal CS distinguished perimitral reentry from other reentries within the LA. When left ATs were suggested, activation mapping of LA was performed. Rove catheters (Biosense Webster, Inc., Irvine, CA, USA) were deployed through the long sheath to acquire stable tissue contact at each location and to create activation maps. Potential critical isthmuses, often containing fractionated electrocardiograms, were identified as areas of constrained activation, resulting from idiopathic or iatrogenic scars and anatomic barriers. Once electrical isolation in all PVs was confirmed and the wave fronts of atrial activation were identified by activation mapping, entrainment-pacing maneuvers were performed from the ablation catheter with a cycle length 20-30 msec less than the tachycardia cycle length. The entrainments were as follows: The first entrainment was performed at the anterior and posterior wall of the LA, then the left atrial ridge, $\mathrm{PV}$, roof and septal area of LA and LA anterior wall (LAAW) near the mitral annulus (Fig. 1). The site was considered part of the circuit if the PPI measured from the stimulation artifact to the return atrial electrocardiogram on the ablation catheter was within $20 \mathrm{msec}$ of the TCL.

Follow-up. Patients were assessed at 3, 6 and 12 months following the procedure and underwent the transthoracic echocardiography and ambulatory monitoring. Postprocedural anticoagulation was continued for $\geq 3$ months in the absence of arrhythmia recurrence or for a longer period otherwise.

Statistical analysis. Data are reported as the mean \pm standard deviation. Comparisons between groups were performed with Student's t-test. $\mathrm{P}<0.05$ was considered to indicate a statistically significant difference.

\section{Results}

Study Population. Sustained ATs within LAA were identified in 3 patients (age range, 63-74; 66\% male) from a cohort of 76 patients undergoing repeated ablation for symptomatic AF. Circumferential PVI was successfully performed in all paroxysmal AF patients during the first procedure. A fixed ablation approach consisting of circumferential PV anteroom 
Table I. Patient characteristics.

\begin{tabular}{lccc}
\hline & $\begin{array}{c}\text { Paroxysmal } \\
\text { AF }\end{array}$ & $\begin{array}{c}\text { Persistent } \\
\text { AF }\end{array}$ & P-value \\
\hline Numbers & 21 & 55 & \\
Male/Female & $14 / 7$ & $30 / 25$ & $>0.05$ \\
Age (years) & $62.1 \pm 9.9$ & $65.3 \pm 8.7$ & $>0.05$ \\
LA diameter (mm) & $42.4 \pm 4.3$ & $47.2 \pm 3.8$ & $>0.05$ \\
EF (\%) & $63.6 \pm 6.2$ & $57.8 \pm 7.3$ & $>0.05$ \\
Presence of structure & 2 & 5 & $>0.05$ \\
heart disease & & & \\
Duration of AF (years) & $3.4 \pm 0.9$ & $4.2 \pm 0.7$ & $>0.05$ \\
\hline
\end{tabular}

AF, atrial fibrillation; LA, left atrium; EF, ejection fraction.

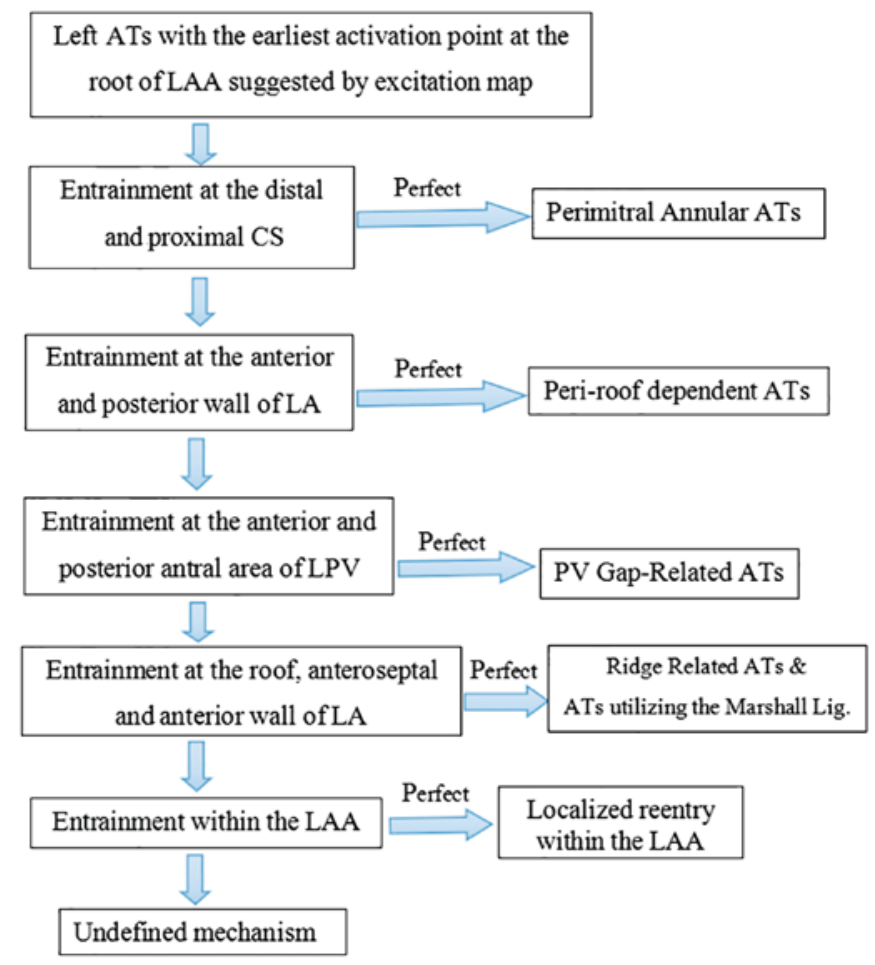

Figure 1. Scheme of entrainment. For patients undergoing ablation for symptomatic atrial fibrillation, activation and entrainment mapping were undertaken as diagnostic techniques to differentiate focal, localized and macro-reentry AT. For rapid distinction of left and right ATs, entrainment was performed at the high RA, proximal and distal CS, respectively. For LA circuits, PPI-TCL difference at the proximal and distal CS distinguished perimitral reentry from other reentries within the LA. When left ATs were indicated, entrainments were as follows: First entrainment was performed at the anterior and posterior wall of the LA, left atrial ridge, PV, roof and septal area of LA and LAAW near the mitral annulus; AT, atrial tachycardia; RA, right atrium; CS, coronary sinus; LA, left atrium; PPI-TCL, postpacing interval-tachycardia cycle length; PV, pulmonary vein; LAAW, left atrial anterior wall; LAA, left atrial appendage; LPV, left PV.

isolation (PVAI) and three linear ablation lesion sets across the mitral isthmus, left atrial roof and cavotricuspid isthmus had been performed in persistent AF, during first time ablation. There was no statistically significant difference in age, sex, AF history, EF, structural heart diseases and LA diameters among the groups as presented in Table I. One patient with previous persistent AF had presented electrical isolation of all four PVs at the time of AT occurrence. All patients undergoing ablation had failed treatments with $\geq 1$ anti-arrhythmic agent.

Arrhythmia characteristics. Macro-reentries including mitral annular, ring gap-dependent, roof-dependent and cavotricuspid isthmus-dependent flutters were observed in this cohort. Mitral annular atrial flutter was reported for $15(19.7 \%)$ patients, and left atrial flutter sustained by reentry through $\geq 2$ gaps in the isolation ring of PV was observed in $6(7.8 \%)$ patients. Typical right atrial flutter, which was anticlockwise around the tricuspid annulus and dependent on the cavotricuspid isthmus, was observed in 2 (2.6\%) patients and an additional 2 patients $(2.6 \%)$ were observed to have a roof dependent flutter. Local reentry within the LAA was recognized in 3 patients $(3.9 \%)$. In $2(2.6 \%)$ patients, the tachycardia occurred spontaneously and in 1 patient (1.3\%), arrhythmia was induced by burst pacing. The mean atrial cycle length of the tachycardia was $264 \pm 15 \mathrm{msec}$ (range $250-280 \mathrm{msec}$ ). The tachycardia demonstrated a characteristic P-wave morphology. The P-wave was highly positive in inferior leads of all patients. Lead V1 displayed upright or biphasic ( \pm ) components. Lead V2-V6 exhibited isoelectric component or upright components with low amplitude. A characteristically high negative P-wave was detected in lead I in all 3 patients with local reentry within the LAA.

Endocardial mapping. Activation mapping was performed in the 3 patients with local reentry within the LAA and the earliest endocardial activation site was at the root of LAA. In 1 patient, due to the unsuccessful ablation of mitral isthmus, an anterior wall ablation line was created (Fig. 2A). During the additional ablation, inadvertent isolation of the LAA was noted, which was confirmed by ATs contained entirely within the LAA, as recorded by lasso catheter (Fig. 2C). Fig. 2B presents an EKG exhibiting a characteristic positive $\mathrm{P}$-wave in the inferior lead and a negative P-wave in lead I and the avL lead. Focal ablations were applied to terminate AT (Fig. 2D). In another patient, distinguishing local reentry from the macro-reentry ATs was affected by the presence of ablation lines (Fig. 3A). AT demonstrated a characteristic P-wave morphology, idicating an LAA origin (Fig. 3B). In addition, entrainment at the base of LAA indicated a localized reentry (Fig. 3C) and ablation at the base of LAA successfully terminated the AT (Fig. 3D). Focal reentry within the LAA in paroxysmal AF was confirmed in 1 patient (Fig. 4A). Local reentry within LAA was confirmed by local entrainment (Fig. 4B). A low-amplitude and long fractionated electrocardiogram was recorded (Fig. 4C), which indicated the critical isthmus of the arrhythmia. Ablation applied at the same time point successfully terminated the arrhythmia that was exhibited (Fig. 4D).

Successful ablation sites. In the present study, ablation was not performed circumferentially and was targeted to the segment of interest in an attempt to avoid severely delayed LAA activation or complete isolation. In each case, the successful ablation site was at the base of LAA with long fractionated or mid-diastolic electrocardiograms and resulted in tachycardia termination. 

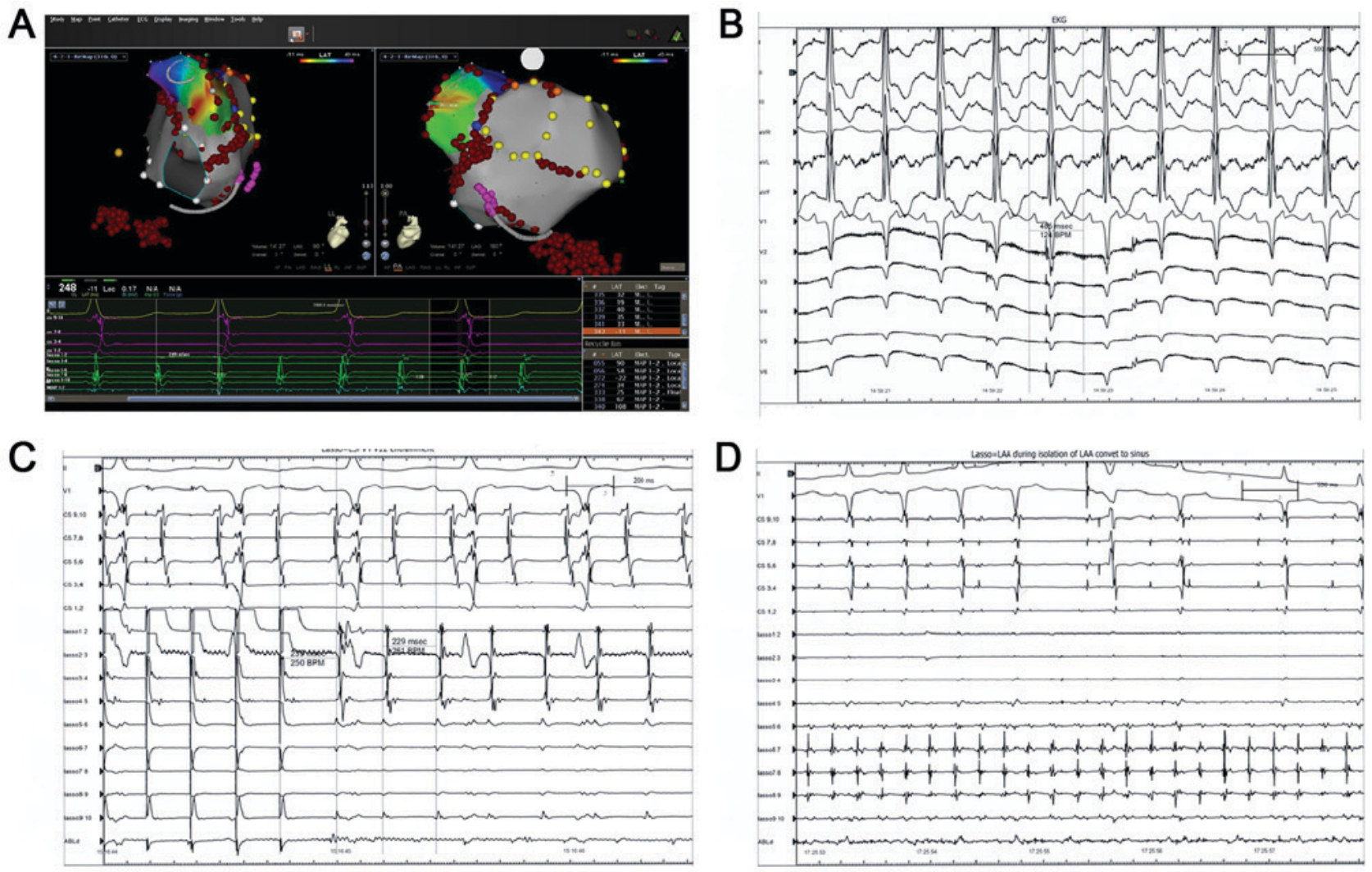

Figure 2. An 80-year-old female patient admitted for repeat RFCA with persistent atrial fibrillation following a fixed ablation approach consisting of circumferential pulmonary vein anteroom isolation and three linear ablation lesion sets across the mitral isthmus, left atrial roof and cavo-tricuspid isthmus. During the ablation of mitral isthmus, the patient converted back to sinus rhythm. As a response to bidirectional mitral isthmus conduction block, an additional ablation line was created at the anterior wall. During this procedure, an organized AT was observed. (A) First endocardial post diastolic activation site was observed at the base of the LAA. (B) EKG displaying a characteristic positive P-wave in inferior lead and negative P-wave in lead I and lead avL. (C) Entrainments were performed at the base of LAA, distal and proximal coronary sinus, roof and anteroseptal parts of left atrium. Tachycardia cycle length was $229 \mathrm{msec}$ and postpacing interval was $250 \mathrm{msec}$ at the base of the LAA, suggesting the pacing site was anatomically adjacent to the circuit. (D) AT completely contained within the LAA as confirmed by lasso catheter. Additional ablation at the base of the LAA eliminated the AT. RFCA, radiofrequency catheter ablation; AT, atrial tachycardia; LAA, left atrial appendage; EKG, electrocardiogram.

\section{Discussion}

It has long been suggested that the potential importance of LAA is not only in triggering, but in long-term maintenance of atrial arrhythmias (25-28). Vazquez et al (29) reported a case with sustained fibrillation contained entirely within the LAA, which continued even following electrical isolation of the LAA. It supported the concept that in patients with AF, LAA isolation should be performed to minimize AF recurrence. ATs following persistent AF ablation are common (7). The most common mechanism of ATs is macro-reentry, which includes mitral annular, ring gap-dependent and roof-dependent flutter $(30,31)$. Other previously described mechanisms include cavotricuspid isthmus-dependent flutters, ridge-associated reentrant ATs and ATs utilizing the ligament of Marshall (31). LAA was suggested as an uncommon site of origin for ATs following AF ablation (18). LAA appears to be responsible for arrhythmias in $27 \%$ of patients who undergo repeated procedures (28). The present study discovered that $4.7 \%$ of patients with paroxysmal AF and $3.6 \%$ of patients with persistent AF had recurrent ATs due to localized reentry within the LAA.

ATs are classified into focal, local reentry and macro-reentry types (32). Activation and entrainment mapping are useful diagnostic techniques in differentiating post-ablation ATs.
Activation mapping is useful for precise positioning and facilitating ablation (33). Activation mapping by itself may not be able to differentiate a focal tachycardia mimicking macro-reentry from a true macro-reentry (34). When tachycardia arises from a focus near a completely blocked ablation line, activation mapping may present an activation sequence similar to macro-reentry. In those cases, additional entrainment mapping is required to differentiate between these types of tachycardia (34). In the current study, a fixed ablation approach was selected, consisting of circumferential PVAI and three linear ablation lesion sets across the mitral isthmus, left atrial roof and cavotricuspid isthmus in persistent AF. Induction of ATs was performed in all AF ablation patients. Following induction of ATs, wave fronts of atrial activation and potential isthmus sites were described using activation maps. The majority of interventions are used to perform entrainment pacing only at the isthmus, as indicated by the activation map, instead of multiple pacing in left atrium to verify the mechanism of ATs. Using this method, the mechanism of certain critical ATs may be misidentified. A sustained AT within the vicinity of LAA occurring following a fixed ablation approach may be an example for this, as it may be mistaken as mitral annual flutter. Therefore, combining activation and entrainment mapping may be important in approaching ATs. 
A

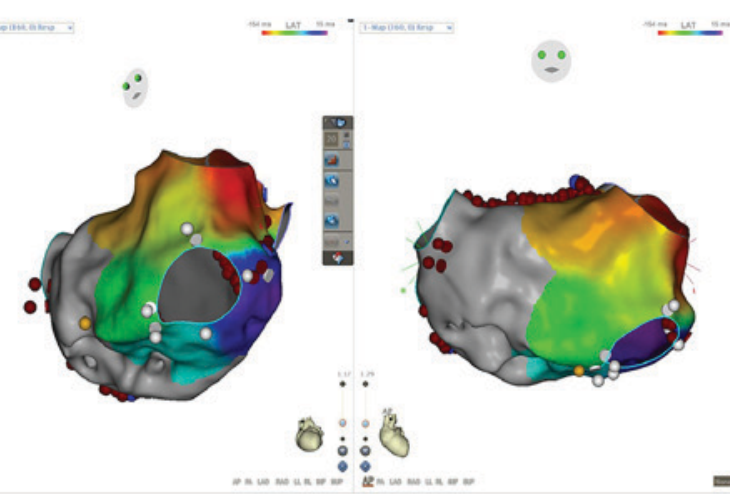

C

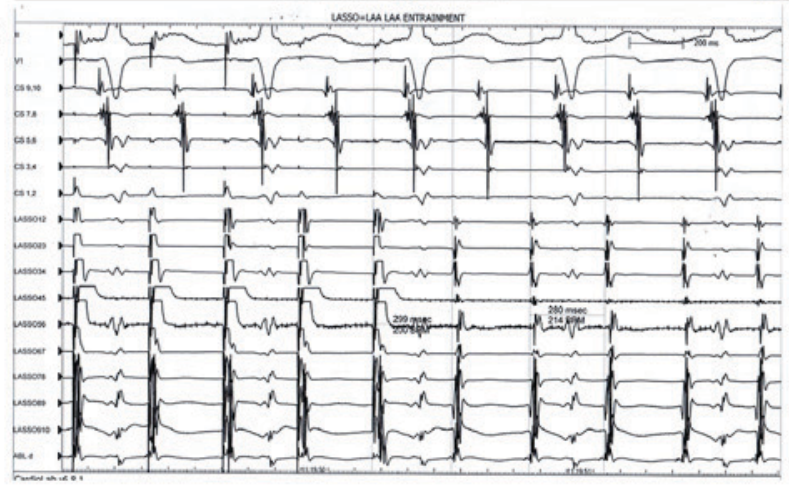

B

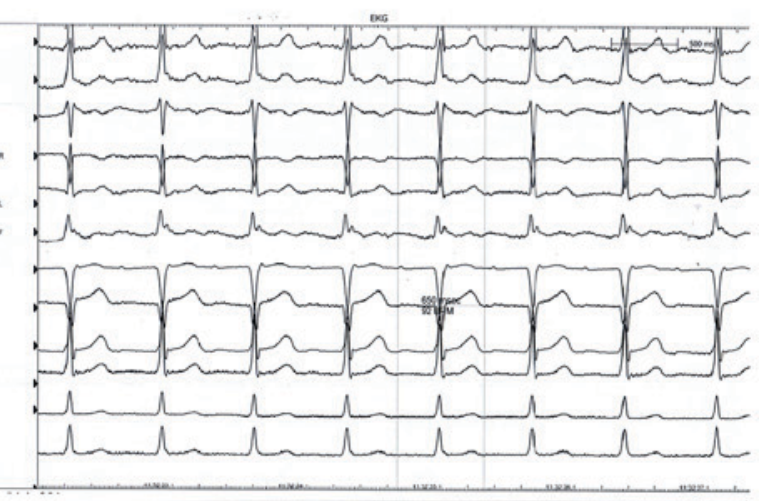

D

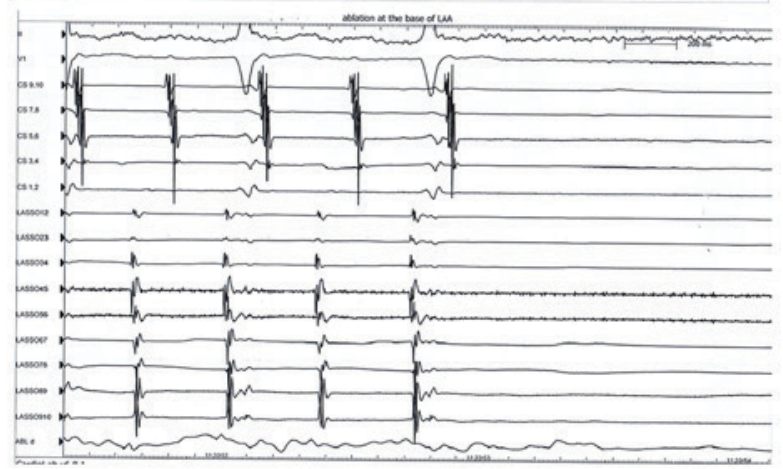

Figure 3. A 67-year-old male patient with persistent atrial fibrillation developed recurrent AT requiring a second procedure following the isolation of pulmonary veins and ablation lesion sets across the mitral isthmus, left atrial roof and cavo-tricuspid isthmus. (A) Activation map mimicking macro-reentry due to the adjacent blocked ablation lines next to the localized reentry at the base of the LAA. (B) AT had a characteristic P-wave morphology that implied an LAA origin. (C) Tachycardia cycle length of AT was $280 \mathrm{msec}$ and postpacing interval was $299 \mathrm{msec}$. (D) Entrainment at the base of LAA suggested a localized reentry. Ablation at the base of LAA successfully terminated the AT. AT, atrial tachycardia; LAA, left atrial appendage.
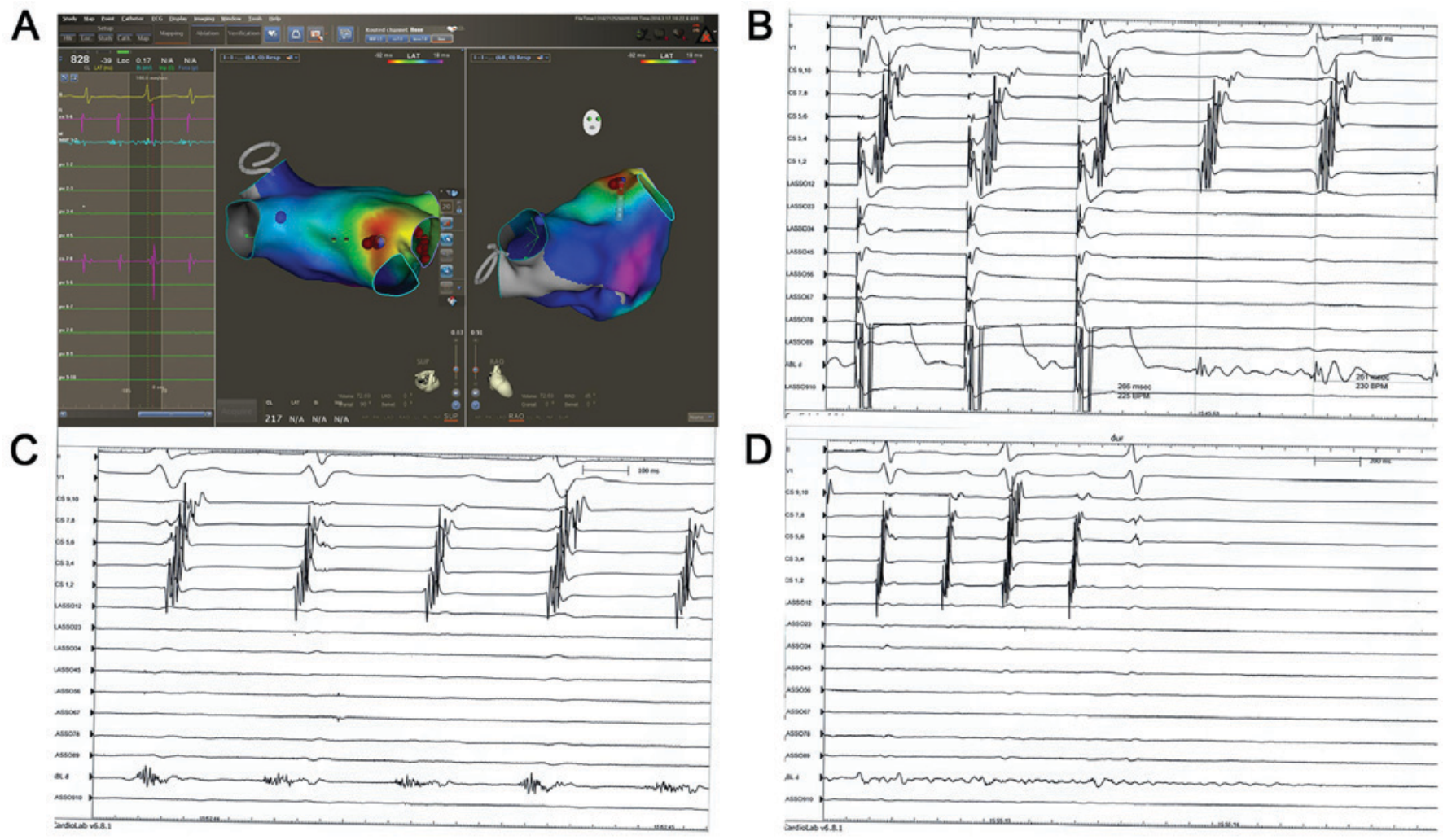

D

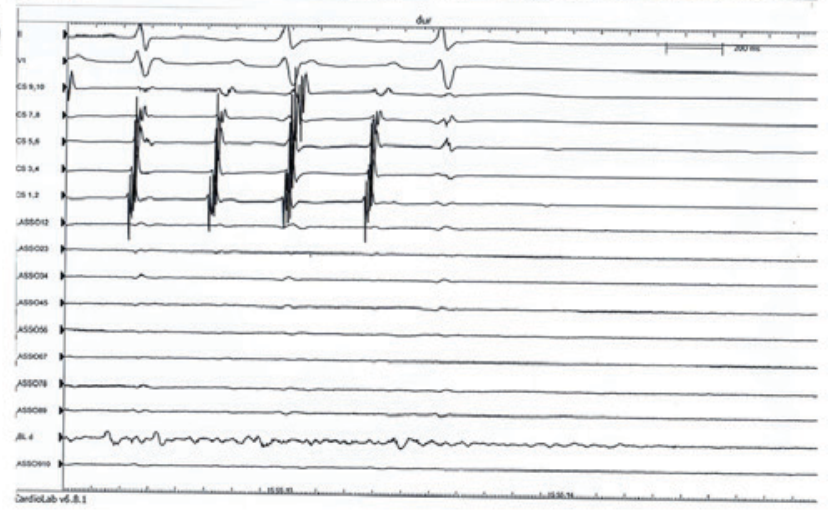

Figure 4. A 41-year-old male patient suffering from paroxysmal atrial fibrillation was readmitted for recurrent palpitations. Paroxysmal AT was induced following isolation of the pulmonary vein during the repeat ablation. (A) Activated map confirming a local reentry mechanism. (B) Entrainment was applied at the base of LAA. (C) Tachycardia cycle length of AT was $261 \mathrm{msec}$ and postpacing interval was $266 \mathrm{msec}$. (D) High-frequency, low-amplitude and long fractionated electrocardiogram was recorded. Ablation applied at that point resulted in tachycardia termination. AT, atrial tachycardia; LAA, left atrial appendage. 
In patients with repeated AF ablation, identification of the atrial wave may prove difficult due to the low voltage of the left atrium. Furthermore, it may lead to a false atrial activation mapping result in cases with local long-range CFAE or unsuccessful setting of the window of interest. In previous studies, mistakes when only applying entrainment mapping of the key isthmus according to the indication of activation mapping were presented (34). A strategy to confirm the results by programmed entrainment mapping may be favorable. The current study demonstrated that AT diagnosed by both activation and entrainment mapping may be verified during ablation.

The mechanism of ATs in the LAA remains unknown; however, it has been demonstrated that the LAA is a remnant of the original embryonic atrium and may be the source of arrhythmia. Iatrogenic ATs have been reported following ablation of either paroxysmal or persistent AF (35). In paroxysmal AF, the mechanism of post-ablation ATs is usually focal and associated to PVs (36). In persistent AF, substrate modification of the left atrium by linear ablation or CFAE ablation may complicate the situation. The post-ablation ATs in persistent AF largely depend on the ablation performed in previous procedures and are variable between cases (37). Anatomic approaches have been reported with a higher prevalence of macro-reentry, while the complex fractionated electrocardiogram ablation strategies may increase the prevalence of focal ATs by up to $50 \%$ of total post-ablation ATs (38). The prevalence of LAA-associated ATs raised the question of whether this type of arrhythmia is a primary change or an iatrogenic origin (22). The current study observed a case of AT located at the vicinity of ablation line surrounding the left ipsilateral PV. Although AT was confirmed within the LAA by activation mapping and left atriogram, there was a possibility that this type of AT was iatrogenic.

To improve the success rates of AF treatment during repeat procedures, Di Biase et al (28) proposed a strategy for the isolation of the LAA rather than focal ablation. Normal contraction of the LAA and adequate blood flow within the LAA may be lost, which increases the risk for the formation of thrombi inside the cavity and impaired function of the LA $(17,39,40)$. In the present study, ablation was not performed circumferentially and was targeted to the segment of interest in an attempt to avoid severely delayed LAA activation or complete isolation. Previous studies were highly effective in acute and medium-term elimination of arrhythmias within the LAA following ablation targeting the site with long fractionated or mid-diastolic LAA electrocardiograms (17). In the present study, no AT recurrence was recorded during follow-up in the 3 patients with local reentry within the LAA following successful ablations.

\section{Acknowledgements}

Not applicable.

\section{Funding}

No funding was received.

\section{Availability of data and materials}

The datasets used and/or analyzed during the current study are available from the corresponding author on reasonable request.

\section{Authors' contributions}

JCG wrote the manuscript and assisted with preoperative preparation. WBH and FGZ performed radiofrequency ablation. JH collected and analyzed the data. YW designed the study. All authors read and approved the final manuscript.

\section{Ethics approval and consent to participate}

Ethics approval for the study was provided by the Institutional Ethics Committee of Xiamen Cardiovascular Hospital (Xiamen, China; XMCH-014-173) and it was in compliance with national legislation and the Declaration of Helsinki guidelines. Patients provided written informed consent.

\section{Consent for publication}

Patient consent was obtained for publication of this manuscript.

\section{Competing interests}

The authors declare that they have no competing interests.

\section{References}

1. Du X, Guo L, He X, Jia Y, Wu J, Long D, Yu R, Sang C, Liu X, Yin $\mathrm{H}$, et al: A comparison of the real world effectiveness of catheter ablation and drug therapy in atrial fibrillation patients in a Chinese setting. BMC Cardiovasc Disord 17: 204, 2017.

2. Haïssaguerre M, Hocini M, Sanders P, Sacher F, Rotter M, Takahashi Y, Rostock T, Hsu LF, Bordachar P, Reuter S, et al: Catheter ablation of long-lasting persistent atrial fibrillation: Clinical outcome and mechanisms of subsequent arrhythmias. J Cardiovasc Electrophysiol 16: 1138-1147, 2005.

3. Zheng L, Yao Y, Zhang S, Chen W, Zhang K, Wang F, Chen X, He DS and Kadish AH: Organized left atrial tachyarrhythmia during stepwise linear ablation for atrial fibrillation. J Cardiovasc Electrophysiol 20: 499-506, 2009.

4. Ju W, Yang B, Chen H, Zhang F, Zhai L, Cao K and Chen M: Localized reentry as a novel type of the proarrhythmic effects of linear ablation in the left atrium. Pacing Clin Electrophysiol 34: 919-926, 2011.

5. Chugh A, Oral H, Lemola K, Hall B, Cheung P, Good E, Tamirisa K, Han J, Bogun F, Pelosi F Jr and Morady F: Prevalence, mechanisms, and clinical significance of macroreentrant atrial tachycardia during and following left atrial ablation for atrial fibrillation. Heart Rhythm 2: 464-471, 2005.

6. Sawhney N, Anand K, Robertson CE, Wurdeman T, Anousheh R and Feld GK: Recovery of mitral isthmus conduction leads to the development of macro-reentrant tachycardia after left atrial linear ablation for atrial fibrillation. Circ Arrhythm Electrophysiol 4: 832-837, 2011.

7. Chae S, Oral H, Good E, Dey S, Wimmer A, Crawford T, Wells D, Sarrazin JF, Chalfoun N, Kuhne M, et al: Atrial tachycardia after circumferential pulmonary vein ablation of atrial fibrillation: Mechanistic insights, results of catheter ablation, and risk factors for recurrence. J Am Coll Cardiol 50: 1781-1787, 2007.

8. Takatsuki S, Fukumoto K, Igawa O, Kimura T, Nishiyama N, Aizawa Y, Tanimoto Y, Tanimoto K, Miyoshi S and Fukuda K: Ridge-related reentry: A variant of perimitral atrial tachycardia. J Cardiovasc Electrophysiol 24: 781-787, 2013.

9. Chik WW, Chan JK, Ross DL, Wagstaff J, Kizana E, Thiagalingam A, Kovoor P and Thomas SP: Atrial tachycardias utilizing the Ligament of Marshall region following single ring pulmonary vein isolation for atrial fibrillation. Pacing Clin Electrophysiol 37: 1149-1158, 2014.

10. Haïssaguerre M, Hocini M, Sanders P, Takahashi Y, Rotter M, Sacher F, Rostock T, Hsu LF, Jonsson A, O'Neill MD, et al: Localized sources maintaining atrial fibrillation organized by prior ablation. Circulation 113: 616-625, 2006. 
11. Yokokawa M, Latchamsetty R, Ghanbari H, Belardi D, Makkar A, Roberts B, Saint-Phard W, Sinno M, Carrigan T, Kennedy R, et al: Characteristics of atrial tachycardia due to small vs large reentrant circuits after ablation of persistent atrial fibrillation. Heart Rhythm 10: 469-476, 2013.

12. Wasmer K, Mönnig G, Bittner A, Dechering D, Zellerhoff S, Milberg P, Köbe J and Eckardt L: Incidence, characteristics, and outcome of left atrial tachycardias after circumferential antral ablation of atrial fibrillation. Heart Rhythm 9: 1660-1666, 2012.

13. Biviano AB, Bain W, Whang W, Leitner J, Dizon J, Hickey K and Garan H: Focal left atrial tachycardias not associated with prior catheter ablation for atrial fibrillation: Clinical and electrophysiological characteristics. Pacing Clin Electrophysiol 35: 17-27, 2012.

14. Kistler PM and Kalman JM: Locating focal atrial tachycardias from P-wave morphology. Heart Rhythm 2: 561-564, 2005.

15. Ho SY, Anderson RH and Sánchez-Quintana D: Atrial structure and fibres: Morphological basis of atrial conduction. Cardiovasc Res 54: 325-336, 2002.

16. Krul SP, Berger WR, Smit NW, van Amersfoorth SC, Driessen AH, van Boven WJ, Fiolet JW, van Ginneken AC, van der Wal AC, de Bakker JM, et al: Atrial fibrosis and conduction slowing in the left atrial appendage of patients undergoing thoracoscopic surgical pulmonary vein isolation for atrial fibrillation. Circ Arrhythm Electrophysiol 8: 288-295, 2015.

17. Hocini M, Shah AJ, Nault I, Sanders P, Wright M, Narayan SM, Takahashi Y, Jaïs P, Matsuo S, Knecht S, et al: Localized reentry within the left atrial appendage: Arrhythmogenic role in patients undergoing ablation of persistent atrial fibrillation. Heart Rhythm 8: 1853-1861, 2011.

18. Feng XF, Lu SB, Wang J and Li YG: Atrial fibrillation arising from the left atrial appendage. Intern Med 54: 3157-3160, 2015.

19. Takahashi Y, Sanders P, Rotter M and Haïssaguerre M: Disconnection of the left atrial appendage for elimination of foci maintaining atrial fibrillation. J Cardiovasc Electrophysiol 16: 917-919, 2005

20. Luther V, Linton NW, Koa-Wing M, Lim PB, Jamil-Copley S, Qureshi N, Ng FS, Hayat S, Whinnett Z, Davies DW, et al: A prospective study of ripple mapping in atrial tachycardias: A novel approach to interpreting activation in low-voltage areas. Circ Arrhythm Electrophysiol 9: e003582, 2016.

21. Schaeffer B and Stevenson WG: Entrainment mapping: Theoretical considerations and practical implementation. J Cardiovasc Electrophysiol 29: 204-213, 2018.

22. Al-Saady NM, Obel OA and Camm AJ: Left atrial appendage: Structure, function, and role in thromboembolism. Heart 82 547-554, 1999

23. Alli O and Holmes D Jr: Evaluation of the WATCHMAN left atrial appendage closure device. Expert Rev Med Devices 11: 541-551, 2014

24. Ho SY and Sánchez-Quintana D: The importance of atrial structure and fibers. Clin Anat 22: 52-63, 2009.

25. Guo XG, Zhang JL, Ma J, Jia YH, Zheng Z, Wang HY, Su X and Zhang S: Management of focal atrial tachycardias originating from the atrial appendage with the combination of radiofrequency catheter ablation and minimally invasive atrial appendectomy. Heart Rhythm 11: 17-25, 2014

26. Yang Q, Ma J, Zhang S, Hu JQ and Liao ZL: Focal atrial tachycardia originating from the distal portion of the left atrial appendage: Characteristics and long-term outcomes of radiofrequency ablation. Europace 14: 254-260, 2012.

27. Rosso R, Morton JB, Aggarwal A and Kalman JM: Image integration to guide ablation of incessant left atrial appendage tachycardia. Heart Rhythm 7: 1913-1914, 2010.
28. Di Biase L, Burkhardt JD, Mohanty P, Sanchez J, Mohanty S, Horton R, Gallinghouse GJ, Bailey SM, Zagrodzky JD, Santangeli P, et al: Left atrial appendage: An underrecognized trigger site of atrial fibrillation. Circulation 122: 109-118, 2010.

29. Vazquez J, B Biviano A, Garan H and Whang W: Sustained fibrillation within the left atrial appendage during catheter ablation for recurrent atrial tachyarrhythmia. J Atrial Fibrillation 5: 581, 2012.

30. Patel AM, d'Avila A, Neuzil P, Kim SJ, Mela T, Singh JP Ruskin JN and Reddy VY: Atrial tachycardia after ablation of persistent atrial fibrillation: Identification of the critical isthmus with a combination of multielectrode activation mapping and targeted entrainment mapping. Circ Arrhythm Electrophysiol 1: 14-22, 2008.

31. Gerstenfeld EP and Marchlinski FE: Mapping and ablation of left atrial tachycardias occurring after atrial fibrillation ablation. Heart Rhythm 4 (3 Suppl): S65-S72, 2007.

32. Lee G, Sanders P and Kalman JM: Catheter ablation of atrial arrhythmias: State of the art. Lancet 380: 1509-1519, 2012.

33. Zhao L, Wu S, Jiang W, Zhou L, Gu J, Wang Y, Liu Y, Zhang X and Liu X: Differential clinical characteristics and prognosis of patients with longstanding persistent atrial fibrillation presenting with recurrent atrial tachycardia versus recurrent atrial fibrillation after first ablation. J Cardiovasc Electrophysiol 25: 259-265, 2014.

34. Ejima K, Shoda M, Miyazaki S, Yashiro B, Wakisaka O, Manaka T and Hagiwara N: Localized reentrant tachycardia in the aorta contiguity region mimicking perimitral atrial flutter in the context of atrial fibrillation ablation. Heart Vessels 28: 546-549, 2013.

35. Nakahara S, Hori Y, Hayashi A, Kobayashi S, Nakamura H, Okumura Y and Takayanagi K: Impact of left atrial appendage ridge ablation on the complex fractionated electrograms in persistent atrial fibrillation. J Interv Card Electrophysiol 41: 55-64, 2014.

36. Lin YJ, Tai CT, Kao T, Tso HW, Higa S, Tsao HM, Chang SL, Hsieh MH and Chen SA: Frequency analysis in different types of paroxysmal atrial fibrillation. J Am Coll Cardiol 47: 1401-1407, 2006.

37. Scherr D, Khairy P, Miyazaki S, Aurillac-Lavignolle V, Pascale $P$ Wilton SB, Ramoul K, Komatsu Y, Roten L, Jadidi A, et al: Five-year outcome of catheter ablation of persistent atrial fibrillation using termination of atrial fibrillation as a procedural endpoint. Circ Arrhythm Electrophysiol 8: 18-24, 2015.

38. Ouyang F, Antz M, Ernst S, Hachiya H, Mavrakis H, Deger FT, Schaumann A, Chun J, Falk P, Hennig D, et al: Recovered pulmonary vein conduction as a dominant factor for recurrent atrial tachyarrhythmias after complete circular isolation of the pulmonary veins: Lessons from double Lasso technique. Circulation 111: 127-135, 2005.

39. Chugh A and Oral H: Delayed activation of the left atrial appendage following catheter ablation of persistent atrial fibrillation: A cause for concern? Pacing Clin Electrophysiol 33: 649-651, 2010

40. Wang YL, Li XB, Quan X, Ma JX, Zhang P, Xu Y, Zhang HC and Guo JH: Focal atrial tachycardia originating from the left atrial appendage: Electrocardiographic and electrophysiologic characterization and long-term outcomes of radiofrequency ablation. J Cardiovasc Electrophysio 18: 459-464, 2007.

This work is licensed under a Creative Commons Attribution-NonCommercial-NoDerivatives 4.0 International (CC BY-NC-ND 4.0) License. 